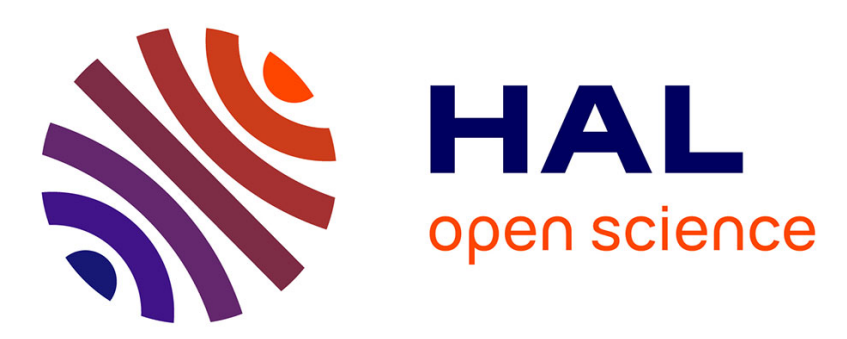

\title{
Estimating the reliability of georeferenced lane markings for map-aided localization
}

\author{
Anthony Welte, Philippe Xu, Philippe Bonnifait, Clément Zinoune
}

\section{To cite this version:}

Anthony Welte, Philippe Xu, Philippe Bonnifait, Clément Zinoune. Estimating the reliability of georeferenced lane markings for map-aided localization. 30th IEEE Intelligent Vehicles Symposium (IV 2019), Jun 2019, Paris, France. pp.1225-1231. hal-02157585

\section{HAL Id: hal-02157585 \\ https://hal.science/hal-02157585}

Submitted on 17 Jun 2019

HAL is a multi-disciplinary open access archive for the deposit and dissemination of scientific research documents, whether they are published or not. The documents may come from teaching and research institutions in France or abroad, or from public or private research centers.
L'archive ouverte pluridisciplinaire HAL, est destinée au dépôt et à la diffusion de documents scientifiques de niveau recherche, publiés ou non, émanant des établissements d'enseignement et de recherche français ou étrangers, des laboratoires publics ou privés. 


\title{
Estimating the reliability of georeferenced lane markings for map-aided localization
}

\author{
Anthony Welte ${ }^{1}$, Philippe $\mathrm{Xu}^{1}$, Philippe Bonnifait ${ }^{1}$, Clement Zinoune ${ }^{2}$
}

\begin{abstract}
Maps can greatly improve vehicle localization using perception sensors that detect features georeferenced in the map. This relies on two assumptions. Firstly, the detected features and the elements of the map have to be correctly associated. Secondly, the features of the map have to be accurately referenced. In this paper, solutions regarding these issues are presented. The case study of localization using a camera detecting road markings is considered. A Kalman smoothing process is used to obtain the best possible estimate of the trajectory that enables to evaluate the reliability of markings stored in the map. A likelihood maximization technique is used to best associate the observed markings to those referenced in the map. By using these two methods, map errors are detected after a first passage in an area and can be mitigated in later passes. Experimental results are reported to evaluate the performance of this approach. It is shown that mapping errors can be correctly handled.
\end{abstract}

\section{INTRODUCTION}

Map-aided localization has been a topic of research in the robotics community for years. Maps can provide valuable information concerning the location of features within the environment [13], [1]. They are particularly relevant in autonomous driving applications since high accuracy is required for such systems. The use of HD maps, maps containing the accurate location of features of the environment, has shown promising results in localization applications [10], [3], [8].

By using perception sensors, features of the map can be detected and used to localize the vehicle. One type of feature particularly interesting for autonomous driving is lane markings. Most roads have lane markings or at least road edges. Several works using LiDAR sensors have demonstrated the interest of detecting such features for localization [4], [3]. More classically, cameras can be used for the same purpose. A significant body of research using camera images exists on the subject [10], [12], [8], [5].

In parallel, sensors such as smart cameras are more and more present on commercial vehicles. These cameras can provide measurements of lane markings for current driving assistance systems such as lane departure warning, or lane keeping in simple road conditions. Using such black-box sensors for localization purposes can be challenging [11]. Indeed the information provided by such cameras is heavily filtered which result in temporally correlated noises.

The methods mentioned above all suffer from two problems. The association of observations to map features can

\footnotetext{
${ }^{1}$ Sorbonne universités, Université de Technologie de Compiègne, CNRS UMR 7253 Heudiasyc, France

${ }^{2}$ Renault S.A.S, Guyancourt, France
}

be difficult when the localization is not accurate. Moreover, ambiguities can occur in the association process especially when detailed maps are used. Another issue is that the map has to be accurate. Even though roads do not change much from one day to the next, they can change when markings are repainted or when road work is done [7], [6]. For that reason, autonomous systems require ways to detect changes in the environment that lead part of the map to become unreliable. Statistical tests have also been used to detect errors in the map [15]. Other works consider the map as imprecise and aim to improve it [2].

In this paper, a method to localize a vehicle using map markings detected by a smart camera is presented. Our main contribution relies on the way to detect and account for map errors using Kalman smoothing and a likelihood maximization based marking association. The map marking locations are modeled using Gaussian distributions and likelihood maximization is used to best match the observations to the map. The map markings can then be associated with each observation and used in a Kalman filter to estimate the current vehicle state.

In section II, the camera observations and the structure of the map is first introduced. Then in section III, our method to use lane markings for localization is presented. Section IV details how the reliability of the markings is computed and taken into account. Finally, section V shows results obtained using an experimental vehicle on public roads.

\section{LANE MARKING OBSERVATIONS AND HD MAP}

\section{A. Lane marking observations}

The camera considered in this paper provides up to four lane marking detections at a time. At every measurement epoch $k$, it provides for each detected marking $j$, a measurement of the distance $C_{k}^{j}$ from the observed marking to the vehicle longitudinal axis as shown in Figure 1.

The lane markings can be considered locally as straight lines. Therefore for each detection, a new lateral constraint can be applied on the state. This constraint is implemented through a Kalman filter estimating the two-dimensional pose $\boldsymbol{x}_{k}=\left[\begin{array}{lll}x_{k} & y_{k} & \psi_{k}\end{array}\right]^{T}$ (East, North, Heading) of the vehicle. For each observation, a simple observation model can be conceived using the following observation matrix:

$$
H_{k}=\left[\begin{array}{lll}
\sin \left(\psi_{k}\right) & -\cos \left(\psi_{k}\right) & 0
\end{array}\right] .
$$

The innovation of the filter $\tilde{\boldsymbol{y}}_{k}$ is then the difference between the observation $C_{k}^{j}$ and the expected observation 


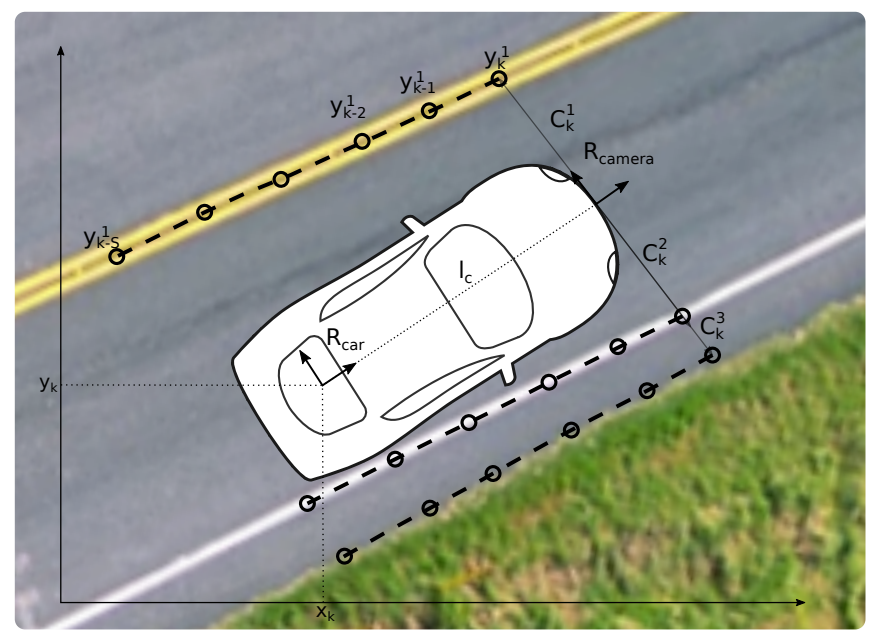

Fig. 1. Schema of the vehicle and the camera measurements. The camera observes both markings and road edges.

$D_{m}\left(\boldsymbol{x}_{k}\right)$ (lateral distance between the state and the associated marking $m$ ),

$$
\tilde{\boldsymbol{y}}_{k}=C_{k}^{j}-D_{m}\left(\boldsymbol{x}_{k}\right) .
$$

The camera tracks and filters the detections internally which results in measurement errors that are correlated from one epoch to the next. To avoid fusing observations with correlated noise, the fusion using the camera measurements is performed at a lower frequency than the observations are received (in our experiments a camera update is only performed every 0.5 second even though the camera provides observations at $36 \mathrm{~Hz}$ ). The frequency has been chosen to ensure that the measurements are not correlated while enabling frequent enough updates to avoid drift. The measurements are saved in a buffer and will be used as a batch to perform the association and the fusion. This is detailed in section III.

\section{B. HD map}

To be able to use the marking observations, the locations of the markings need to be known. For that purpose, a map that accurately references the location of markings in the experimentation area is used. The markings are defined as a series of points forming a polyline. The map also stores road edges and curbs. This makes associating observations with the map marking harder since these elements are usually close to each other and can be misclassified by the camera.

\section{LOCALIZATION USING LANE MARKINGS}

\section{A. Observations and map association}

Associating an observed marking with a map marking is often done using the smallest distance between observation and map [10], [11]. This method is very reliant on the accuracy of the state estimate. This increasingly becomes an issue as maps become more detailed, increasing the number of potential matches and therefore the matching ambiguities.

For that reason, the association is performed in two steps. The first step is to find the transformation to apply to the

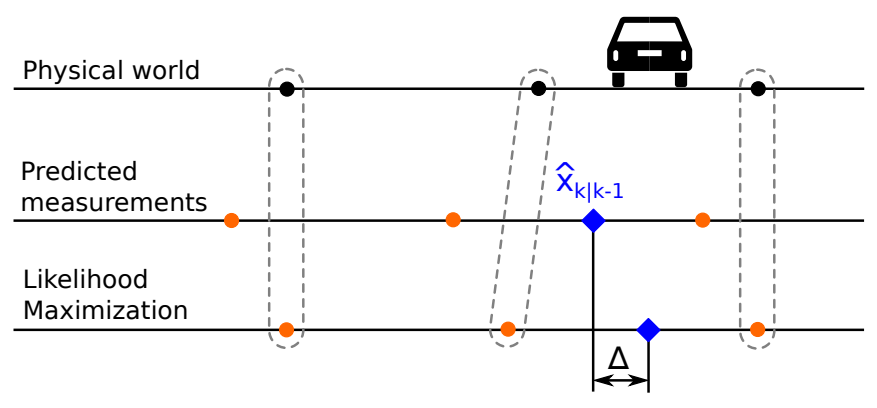

Fig. 2. Cross-section representing the steps used to associate observations with markings. The top row illustrates the map and the true vehicle position. The middle row shows the measurement position relative to the state. The last row shows the result after likelihood maximization where the associations (dashed lines) are selected.

observations so that they best overlap the map. Then, the observations can be associated with a marking by finding the closest marking after the transformation. This process is illustrated in Figure 2.

1) Lane markings observations: As mentioned previously, the marking observations are saved in a buffer and used as a batch. Therefore, at epoch $k$, when the camera observations are used in the filter, a set of observations $\left\{C_{i}^{j}\right\}_{j \in t r a c k s, i \in \llbracket k-S, k \rrbracket}$ is available, where tracks is the list of tracks observed since the last camera update and $S$ is the size of the buffer.

The first step is to convert these measurements to points using the estimated state of the vehicle. For each measurement, the two-dimensional points they correspond to are computed in the global frame using the following equation:

$$
\left\{\begin{array}{l}
X_{i}^{j}=\hat{x}_{i}+l_{c} \cdot \cos \left(\hat{\psi}_{i}\right)-C_{i}^{j} \cdot \sin \left(\hat{\psi}_{i}\right) \\
Y_{i}^{j}=\hat{y}_{i}+l_{c} \cdot \sin \left(\hat{\psi}_{i}\right)+C_{i}^{j} \cdot \cos \left(\hat{\psi}_{i}\right)
\end{array},\right.
$$

where $l_{c}$ is the distance between the vehicle frame and the camera frame, and $\hat{x}_{i}, \hat{y}_{i}, \hat{\psi}_{i}$ are respectively the estimated east and north coordinates and heading at the time $i$ of the observations.

We therefore have a list of points
$\left\{\left[\begin{array}{ll}X_{i}^{j} & Y_{i}^{j}\end{array}\right]^{T}\right\}_{j \in \text { tracks }, i \in \llbracket k-S, k \rrbracket}$ corresponding to detections of multiple lane markings. These points are relative to the global reference frame. To simplify the rest of the computation, both the map and the observed points are moved into the vehicle frame which is positioned and oriented based on the predicted state $\hat{\boldsymbol{x}}_{k \mid k-1}$ (state at which the estimation using the lanes observation is performed). In the rest of this section, the observations in this local frame are referenced using lower-case letters, $\left.\left\{\begin{array}{ll}x_{i}^{j} & y_{i}^{j}\end{array}\right]^{T}\right\}_{j \in \text { tracks }, i \in \llbracket k-S, k \rrbracket}$.

2) Transformation definition: To correctly associate the observations to the map markings, an adjustment step is first performed to best overlap the measurements and the map. Since a two-dimensional case is considered, to find the overlap three components have to be estimated: a twodimensional translation and a rotation. However, since lane markings can only be properly detected in relatively straight 
lines, the longitudinal translation is hard to estimate. Hence, it is assumed that it is null. Also, in this work, the estimation of the vehicle heading is assumed to be fairly good since the evolution model used is well calibrated [14]. Therefore, the rotational component will not be estimated. Finally, only the lateral shift to apply has to be found. Since the local frame of the vehicle is used, it is the shift along the $y$ axis that needs to be estimated.

3) Map modeling: To find the transformation $\Delta$ that makes the observed points and the map best overlap, the value $\Delta$ that maximizes the likelihood of obtaining the set of points $\left\{\left[\begin{array}{ll}x_{i}^{j} & y_{i}^{j}+\Delta\end{array}\right]^{T}\right\}_{j \in \text { tracks }, i \in \llbracket k-S, k \rrbracket}$ with the set of lane markings $\{m\}_{m \in \text { map }}$ is searched for.

The likelihood of measuring a point at the coordinates $\left[\begin{array}{ll}x_{i}^{j} & y_{i}^{j}\end{array}\right]^{T}$ given a map (loaded around the vehicle to limit its size) composed of $M$ markings is

$$
\begin{aligned}
& f\left(\left[\begin{array}{l}
x_{i}^{j} \\
y_{i}^{j}
\end{array}\right] \mid \text { map }\right)= \\
& f\left(\left[\begin{array}{l}
x_{i}^{j} \\
y_{i}^{j}
\end{array}\right] \mid \text { map, },\left[\begin{array}{c}
x_{i}^{j} \\
y_{i}^{j}
\end{array}\right] \leftrightarrow m_{1}\right) f\left(\left[\begin{array}{c}
x_{i}^{j} \\
y_{i}^{j}
\end{array}\right] \leftrightarrow m_{1}\right) \\
& +\ldots+ \\
& f\left(\left[\begin{array}{c}
x_{i}^{j} \\
y_{i}^{j}
\end{array}\right] \mid \operatorname{map},\left[\begin{array}{c}
x_{i}^{j} \\
y_{i}^{j}
\end{array}\right] \leftrightarrow m_{M}\right) f\left(\left[\begin{array}{c}
x_{i}^{j} \\
y_{i}^{j}
\end{array}\right] \leftrightarrow m_{M}\right) \\
& +C,
\end{aligned}
$$

where $\left[\begin{array}{ll}x_{i}^{j} & y_{i}^{j}\end{array}\right]^{T} \leftrightarrow m$ means that the observation $\left[\begin{array}{ll}x_{i}^{j} & y_{i}^{j}\end{array}\right]^{T}$ corresponds to the marking $m$, and $C$ is a constant used to account for the case where the observation does not correspond to any known marking.

Since no prior information is available as to which marking a particular observation is associated with, $f\left(\left[\begin{array}{ll}x_{i}^{j} & y_{i}^{j}\end{array}\right]^{T} \leftrightarrow m\right)$ is considered identical for each observation and marking and equal to $1 / M+1(C$ is also taken equal to $1 / M+1)$.

$f\left(\left[\begin{array}{c}x_{i}^{j}{ }^{j} \\ y_{i}^{j}+\Delta\end{array}\right] \mid \operatorname{map},\left[\begin{array}{c}x_{i}^{j} \\ y_{i}^{j}+\Delta\end{array}\right] \leftrightarrow m\right)$ is ${ }^{\text {In }}+\Delta$ thitten as $L_{i, j, m}(\Delta)$ to lighten equations. $L_{i, j, m}$ is modeled as a Gaussian distribution centered on the marking and spreading along the $y$ axis. Hence, for a marking $m$ the likelihood of measuring the point $\left[\begin{array}{ll}x_{i}^{j} & y_{i}^{j}+\Delta\end{array}\right]^{T}$ associated with the marking $m$ is

$$
L_{i, j, m}(\Delta)=\frac{1}{\sqrt{2 \pi \sigma^{2}}} \exp \left(-\frac{\left(y_{i}^{j}+\Delta-{ }^{m} y_{i}^{j}\right)^{2}}{2 \sigma^{2}}\right),
$$

where ${ }^{m} y_{i}^{j}$ is the projection along the $y$ axis of the observation $\left[\begin{array}{ll}x_{i}^{j} & y_{i}^{j}\end{array}\right]^{T}$ on the marking $m$ (this is illustrated in Figure 3), and $\sigma^{2}$ is the variance representing the combined uncertainty of the marking $\left(\sigma_{m}^{2}\right)$, the measurement $\left(\sigma_{i, j}^{2}\right)$, and estimated state $\left(\sigma_{\text {lat }}^{2}\right)$, see equation (6).

$$
\sigma^{2}=\sigma_{m}^{2}+\sigma_{i, j}^{2}+\sigma_{l a t}^{2} .
$$

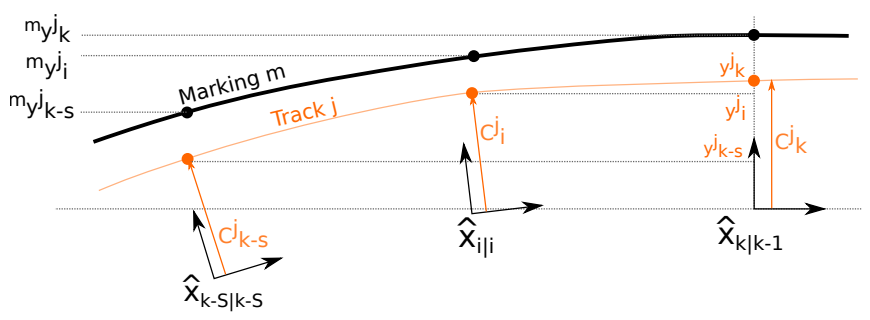

Fig. 3. Representation of the observation buffer and its projection on the lane marking $m$. In orange is displayed the observed track $j$, in black the map marking and projections of the observations.

The map variance $\sigma_{m}^{2}$ corresponds to the uncertainty of the marking considered (equal to 0 since all markings are initially assumed as correct), $\sigma_{i, j}^{2}$ stands for the uncertainty of the measurement and $\sigma_{\text {lat }}^{2}$ is the lateral uncertainty of the state estimate.

The likelihood of having a lane marking detection at the point $\left[\begin{array}{ll}x_{i}^{j} & y_{i}^{j}\end{array}\right]^{T}$ with a map having multiple lane markings is

$$
L_{i, j}(\Delta)=\frac{1}{M+1}\left(\sum_{m \in m a p} L_{i, j, m}(\Delta)+1\right) .
$$

Therefore, the likelihood of detecting the list of points $\left\{\left[\begin{array}{c}x_{i}^{j} \\ y_{i}^{j}+\Delta\end{array}\right]\right\}_{j \in \text { tracks }, i \in \llbracket k-S, k \rrbracket}$ with the lane markings contained in map is

$$
\begin{aligned}
L(\Delta) & =\prod_{\substack{j \in \text { tracks, } \\
i \in \llbracket k-S, k \rrbracket}} L_{i, j}(\Delta) \\
& =\prod_{\substack{j \in \text { tracks, } \\
i \in \llbracket k-S, k \rrbracket}}\left(\frac{1}{M+1}\left(\sum_{m \in \text { map }} L_{i, j, m}(\Delta)+1\right)\right) .
\end{aligned}
$$

The best transformation corresponds to the value $\Delta$ that maximizes the log-likelihood, thus

$$
\begin{aligned}
\hat{\Delta} & =\arg \max _{\Delta}(\log (L(\Delta))) \\
& =\arg \max _{\Delta}\left(\sum_{\substack{j \in \text { tracks, } \\
i \in \llbracket k-S, k \rrbracket}} \log \left(L_{i, j}(\Delta)\right)\right) .
\end{aligned}
$$

4) Likelihood maximization: To solve this problem a gradient descent technique is used. The maximum of the function $\sum_{\substack{j \in \text { tracks } \\ i \in \llbracket k-S, k \rrbracket}} \log \left(L_{i, j}(\Delta)\right)$ is found by iteratively following the direction of its gradient. This method converges after a few iterations, and so more advanced resolution techniques are unnecessary. The transformation $\Delta$ is initialized at 0 . At each iteration, $\Delta$ is increased by

$$
\gamma \cdot \frac{d(\log L)}{d \Delta}(\Delta)=\gamma \times \sum_{\substack{j \in \text { tracks, } \\ i \in \llbracket k-S, k \rrbracket}} \frac{\sum_{m \in \text { map }} \frac{d L_{i, j, m}}{d \Delta}(\Delta)}{\sum_{m \in \text { map }} L_{i, j, m}(\Delta)+1}
$$



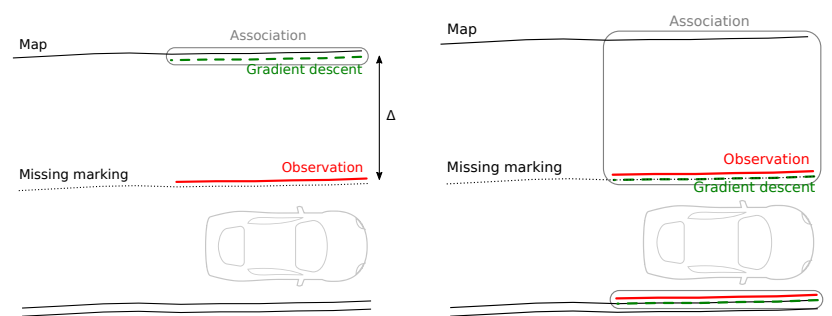

(a) The maximization converges on (b) The maximization converges the wrong association because of a properly but the closest marking does missing marking. not match the observation.

Fig. 4. Graphical representation of cases requiring an outliers rejection step.

where $\gamma$ is chosen empirically. This iterative process stops when the update from an iteration to the next is smaller than a given threshold $\eta$ or when the process reaches a given maximum number of iterations.

5) Association: Once $\hat{\Delta}$ has been found, each track can be associated with a marking. To do so, for each track, the likelihood that it matched a marking $m$ is computed as

$$
L_{j, m}=\prod_{i \in \llbracket k-S, k \rrbracket} L_{i, j, m}(\hat{\Delta}) .
$$

The track $j$ is associated to the marking for which this likelihood is maximum. Therefore, the track $j$ is associated to the marking

$$
\begin{aligned}
m & =\arg \max _{m} L_{j, m} \\
& =\arg \max _{m} \prod_{i \in \llbracket k-S, k \rrbracket} L_{i, j, m}(\hat{\Delta}) .
\end{aligned}
$$

6) Outliers rejection: The associations found previously are not directly used to update the state. Several outlier rejection steps are performed. Indeed, missing markings in the map, missing observations or wrong observations can create scenarios where the associations found previously are not accurate.

In particular, in situations where the road only has a central marking but the map has only the road edges referenced, the camera will be able to detect the unreferenced marking. Because the type of marking detected is not reliable enough, the referenced road edges have to be considered as potential association candidates. The likelihood maximization will converge to overlap the detected central marking with the mapped road edges (see Figure 4a). This can be easily solved by discarding every association where $\Delta$ is too high. In the current implementation of the method, the threshold to discard the markings is taken as a constant (the threshold used in our experimentation is given in Table I):

$$
|\Delta|>\delta
$$

Also, the previous method will result in every track being associated with a marking regardless of their distance to one another after the gradient descent. Since multiple markings can be observed at once, the likelihood maximization can accurately converge even if one marking has no correct association. The method will converge so that most tracks overlap a marking but it can happen that some observations do not overlap any map markings either because the marking is missing in the map or the camera detections are erroneous (see Figure 4b). To account for this, a second threshold is used to remove individual tracks if their residual $r_{j}$ (compensated for $\Delta$ ) is too high:

$$
\begin{aligned}
& \left|r_{j}(\Delta)\right|>\epsilon \\
& \left|\frac{1}{S} \sum_{i \in \llbracket k-S, k \rrbracket}\left(y_{i}^{j}+\Delta-{ }^{m} y_{i}^{j}\right)\right|>\epsilon
\end{aligned}
$$

7) Filtering: Now that the association between track and map marking has been done and outliers have been rejected, we can use these measurements in a Kalman filtering scheme as explained in subsection II-A (a more detailed description of the filtering scheme can be found in [14]). Here, the measurements are the values $y_{i}^{j}$ of each track and the measurements a priori are their projections ${ }^{m} y_{i}^{j}$. The innovation used in the filter for a track $j$ can therefore be computed as

$$
\tilde{\boldsymbol{y}}_{k}^{j}=\frac{1}{S} \sum_{i \in \llbracket k-S, k \rrbracket}\left(y_{i}^{j}-{ }^{m} y_{i}^{j}\right) .
$$

The measurement uncertainty of the camera has been estimated by computing the observed marking position using the ground truth and associating it to the closest map marking (in the limit of 0.5 meter). Through this analysis we concluded that the measurement uncertainty of the camera can be modeled by a zero-mean Gaussian distribution with variance $\sigma_{i, j}^{2}=\left(0.1 \cdot y_{i}^{j}\right)^{2}$ (in $m^{2}$ ). The noise model used in the filter also needs to account for the mapping uncertainty. The total variance considered in the estimator is therefore,

$$
\sigma^{2}=\sigma_{m}^{2}+\sigma_{i, j}^{2}
$$

\section{MAP RELIABILITY ESTIMATION}

Even though HD maps are supposed to contain data with centimeter level accuracy, that can only be guaranteed the day of the mapping. Indeed, lane markings can be redrawn and road work can temporarily affect the lane boundaries [7], [6]. This may lead to a slight shift of the markings location. This result with observation residuals that are not sufficiently high to be detected as outliers and lead to a bias in the filtering.

For that reason, it is essential to have a way to detect changes in the map and be able to account for them. This section describes our approach to solve this issue.

In order to evaluate the lane markings reliability, the observation residuals are used. This approach is only viable if the state of the vehicle is estimated accurately, so that the residuals are mainly affected by bad mapping or observations. To do so a Kalman smoothing approach is used.

\section{A. Smoothing}

First, the state of the vehicle has to be accurately estimated. To achieve this, a Kalman smoothing technique [9] is 
used. During the Kalman filtering process the filtered states $\left\{\hat{\boldsymbol{x}}_{k \mid k}\right\}_{k \in \llbracket 0, N \rrbracket}$ and covariance matrices $\left\{P_{k \mid k}\right\}_{k \in \llbracket 0, N \rrbracket}$, as well as the predicted states $\left\{\hat{\boldsymbol{x}}_{k \mid k-1}\right\}_{k \in \llbracket 1, N \rrbracket}$ and covariance matrices $\left\{P_{k \mid k-1}\right\}_{k \in \llbracket 1, N \rrbracket}$ are recorded to be used for the smoothing (the observed tracks and their associated map markings are also recorded to compute the residuals). The smoothed states $\left\{\hat{\boldsymbol{x}}_{k \mid N}\right\}_{k \in \llbracket 0, N \rrbracket}$ can then be computed from epoch $N-1$ to 0 as such,

$$
\begin{aligned}
\hat{\boldsymbol{x}}_{k \mid N} & =\hat{\boldsymbol{x}}_{k \mid k}+\boldsymbol{J}_{k}\left(\hat{\boldsymbol{x}}_{k+1 \mid N}-\hat{\boldsymbol{x}}_{k+1 \mid k}\right), \\
\boldsymbol{P}_{k \mid N} & =\boldsymbol{P}_{k \mid k}+\boldsymbol{J}_{k}\left(\boldsymbol{P}_{k+1 \mid N}-\boldsymbol{P}_{k+1 \mid k}\right) \boldsymbol{J}_{k}^{T},
\end{aligned}
$$

where

$$
\boldsymbol{J}_{k}=\boldsymbol{P}_{k \mid k} \boldsymbol{F}_{k+1}^{T} \boldsymbol{P}_{k+1 \mid k}^{-1} .
$$

and $\boldsymbol{F}_{k}$ is the evolution matrix of the system.

Using the smoothed states $\left\{\hat{\boldsymbol{x}}_{k \mid N}\right\}_{k \in \llbracket 0, N \rrbracket}$ the lane observation points $\left\{\left[\begin{array}{cc}x_{i}^{j} & y_{i}^{j}\end{array}\right]\right\}_{j \in \text { tracks }, i \in \llbracket k-S, k \rrbracket}$ are computed for every observation of every track. The residuals $r_{j}(0)$ are then computed and stored in individual arrays for each map marking. For each marking id $m$, a list of residuals $\left\{r_{j}\right\}_{j \in \llbracket 0, M_{m} \rrbracket}$ is therefore obtained (where $M_{m}$ is the number of tracks associated to marking $m$ ).

\section{B. Reliability evaluation}

To account for a map marking inaccuracy, its weight in the filtering process is changed by adapting its variance. The variance used for a map marking in equation (6) and equation (19) is $\sigma_{m}^{2}$. When no information on a marking exists, it is assumed accurate and has a variance of 0.0 . If the marking is found to be unreliable it will instead be attributed a variance of $\sigma_{b a d}^{2}$ (chosen at $1 \mathrm{~m}^{2}$ ). The variance of a marking varies between these two values based on a factor $p_{m} \in[0,1]$ as follows:

$$
\sigma^{2}=p_{m} \cdot \sigma_{m}^{2}+\left(1-p_{m}\right) \cdot \sigma_{b a d}^{2}+\sigma_{i}^{2} .
$$

The factor $p_{m}$ is chosen so that when $\overline{r_{j}^{2}}=0.0$, the factor tends to 1 and when $\overline{r_{j}^{2}} \rightarrow+\infty$, the factor tends to 0.0 as illustrated in Figure 5. An exponential function is chosen for this purpose:

$$
p_{m}=\exp \left(-\frac{\overline{r_{j}^{2}}}{\alpha^{2}}\right)
$$

where $\alpha$ is a tuning parameter affecting the size of the errors to consider.

\section{EXPERIMENTAL RESULTS}

\section{A. Experimental setup}

Our approach has been evaluated using an experimental vehicle (Renault ZOE) of the laboratory. The vehicle was equipped with a Novatel SPAN-CPT system which computed RTK positioning loosely coupled with a high accuracy IMU. This system is used as ground truth to evaluate our approach. Through the vehicle CAN bus were available: four wheel encoders, a gyro and the steering wheel angle. These were used for the odometric estimation [14]. The vehicle was

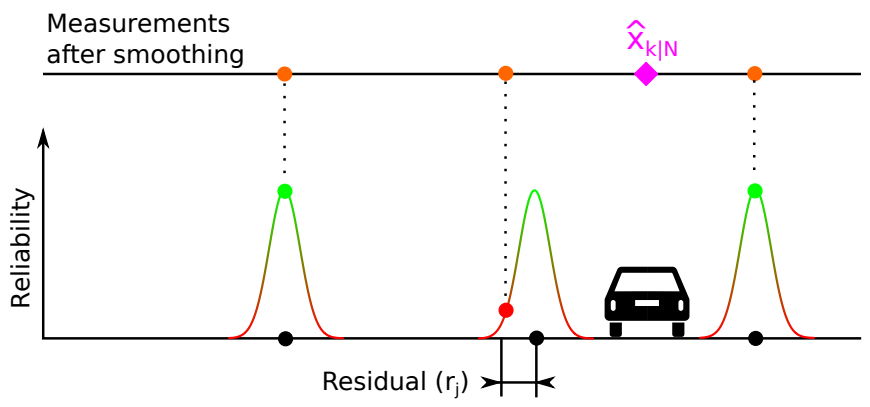

Fig. 5. Attribution of the reliability $p_{m}$ based on $\overline{r_{j}^{2}}$. The top row shows the observations (orange dots) relative to the smoothed state. The residuals between observations and the map (black dots) are used to compute the reliability.

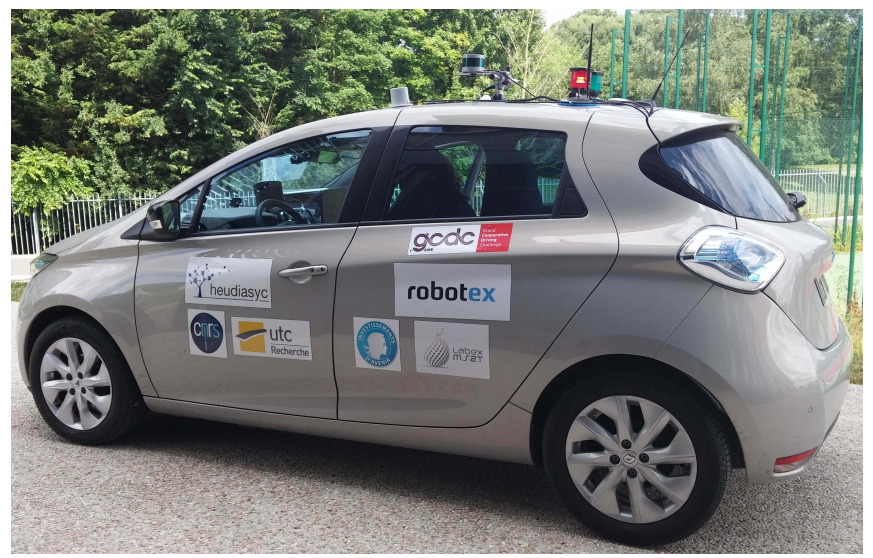

Fig. 6. Experimental vehicle of the Heudiasyc laboratory used in the experiments.

also equipped with a Mobileye EyeQ3 smart camera which provided up to four lane marking observations at a time. The sensors were interfaced using the $\operatorname{ROS}^{1}$ framework. It is the recording and replay feature of this framework that has been used in the following tests.

The experiments were conducted in the city of Rambouillet, France. The path along which the algorithm has been tested is $2 \mathrm{~km}$ long and includes several straight lines and intersections including roundabouts. Moreover, it contains a $200 \mathrm{~m}$ long section where the central map marking is inaccurately localized. Two recordings have been done following this path: one in the morning and one in the afternoon, under similar lighting conditions.

The buffer size chosen for these experiments is 0.5 second, the other parameters of the algorithm are given in Table I.

${ }^{1}$ Robot Operating System: http: //www.ros.org/

TABLE I

PARAMETERS USED IN THE ALGORITHM

\begin{tabular}{|c|c|c|c|c|}
\hline$\gamma$ & $\delta$ & $\epsilon$ & $\eta$ & $\alpha$ \\
\hline 0.2 (unitless) & $1.0 \mathrm{~m}$ & $0.5 \mathrm{~m}$ & $0.001 \mathrm{~m}$ & $0.3 \mathrm{~m}$ \\
\hline
\end{tabular}


TABLE II

NUMBER OF WRONG AND CORRECT ASSOCIATIONS

\begin{tabular}{|c|c|c|c|}
\hline & Wrong & Correct & Proportion \\
\hline Our method & 66 & 133 & $67 \%$ \\
\hline Without finding the overlap & 84 & 112 & $57 \%$ \\
\hline
\end{tabular}

\section{B. Association between observations and map markings}

To evaluate our method for associating observations with lane markings, the associations using the ground truth state, based on closest distance have been used as true associations. The same was done without the likelihood maximization step to provide a point of comparison. Only the results for the associations that have not been rejected in the outliers rejection step are considered.

This test is performed on a particularly challenging section of road. Both on the right and left side of the road, several markings are referenced in the map separated by less than 0.3 meter. The results are presented in Table II.

The distance between markings in the map having the same order of magnitude as the sensor noise, the number of failed association is quite high in both cases. The results, however, show that using likelihood maximization, the matching accuracy is improved in such difficult situations.

\section{Reliability evaluation}

The reliability of the map markings has been evaluated using the first recording. Three markings of the map used in these experiments are known to be incorrectly located. This error is on a straight road oriented north-south and concerns the marking separating both lanes. The actual marking is located roughly 0.5 meter west of the mapped marking. To our knowledge, this is the only marking that is erroneous along the testing path.

Some GNSS measurements are added to the filter every second to avoid drift in intersections. This enables us to complete the full trajectory without drifting out and therefore been able to evaluate the markings using data going to the end of the trajectory and coming back to the initial position.

The result of the reliability evaluation shows that most markings have been assigned a reliability close to 1 . Also the erroneous markings have been correctly assigned a reliability close to 0 as Figure 7 shows. The histogram of the values of $p_{m}$ is given in Figure 8. It can be seen that on the overall trajectory several other map markings have been attributed a low reliability. This can be explained either by sensor errors or by the small number of samples used, in particular on higher speed roads.

\section{Localization}

The accuracy of the localization is evaluated using the second recording. To evaluate the method, the ROS framework is used. The sensor data have been recorded in Rambouillet and can be replayed to test a program. The replay feature of ROS is not perfect. In particular, the small timing differences can appear and slightly affect the final result. This is due to

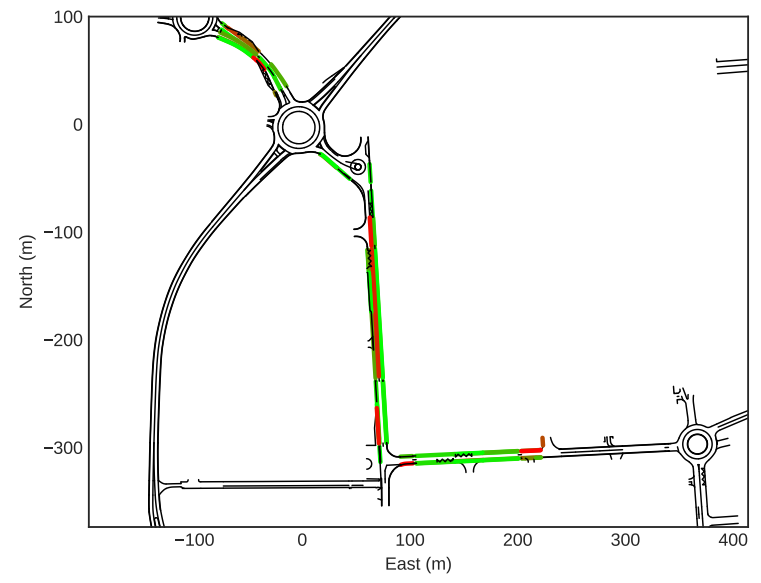

Fig. 7. Representation of marking reliability. (black uncategorized, green most reliable, red least reliable)

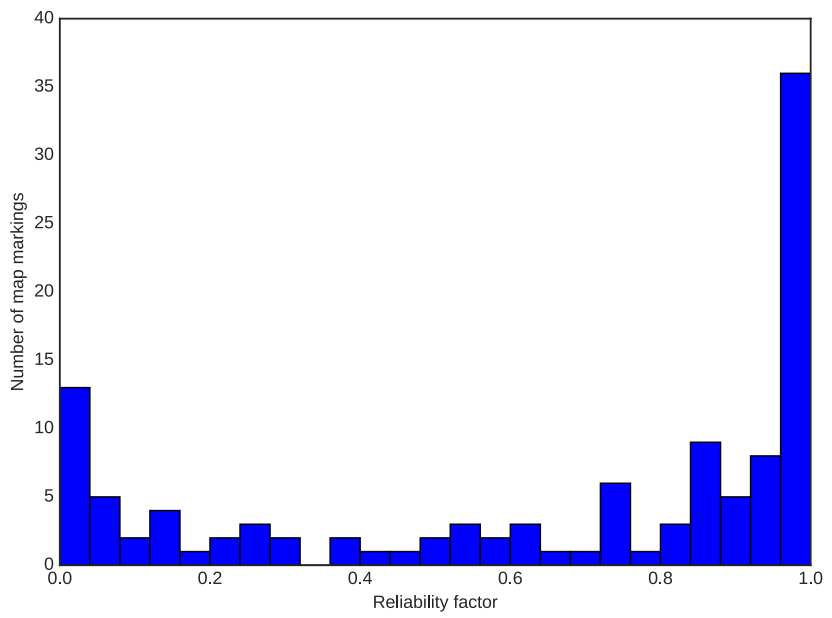

Fig. 8. Distribution of the reliability factor $p_{m}$.

the way ROS handles the transmission of messages between programs. It uses a network based architecture which makes it prone to transmission delays. Hence, a program tested with the same data several times will not produce the exact same result. This could make it hard to interpret the results. To circumvent this issue, the method using the reliability factor and the method that does not have been run multiple times with the same dataset. The result of each run is averaged to limit the effect of the timing differences. The final results are presented in Figure 9.

For these tests, no GNSS was used in the estimation process. This has been done to avoid that the GNSS measurements compensate for the error in the map and mask the effect of using the reliability factor. The system, therefore, estimates the position using only the dead-reckoning sensors and the lane markings measurements. The system has been evaluated on the portion of the trajectory before the first roundabout since without GNSS it sometimes drifts out of it. 


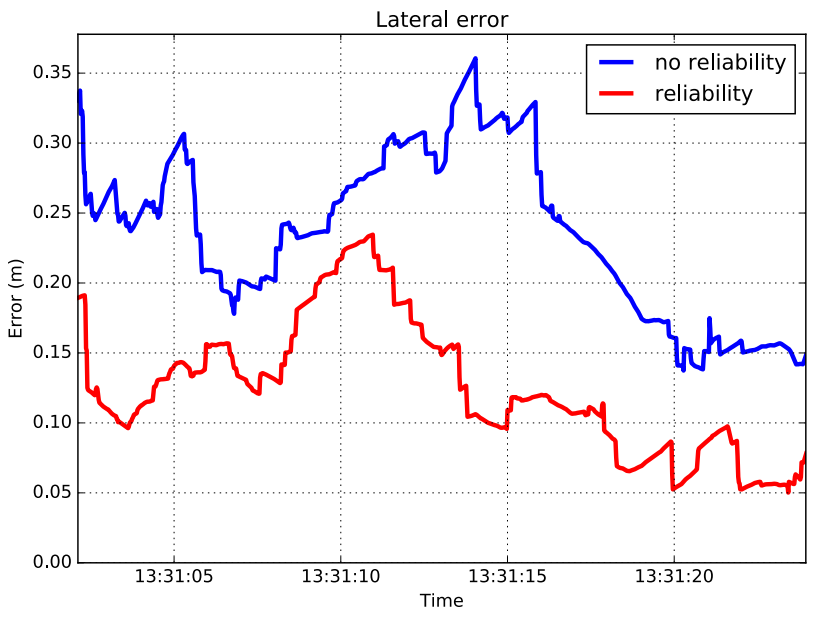

Fig. 9. Localization cross-track error on a section of road where the map is erroneous. The blue line is the error of an estimation assuming every marking are equally accurate. The red line is the result of an estimation weighting the markings based on the reliability factor.

The results show an improvement in the lateral localization accuracy when using the reliability factor of about 0.12 meter on average while traversing the area with the map error (see Figure 9).

Also in the area where the map has no error the result of using or not a reliability factor yields similar results.

\section{CONCLUSION}

In this paper, a method to estimate the reliability of map markings has been proposed. This enables to have localization robust to erroneous mapping. Lateral accuracy of less than 0.2 meter even with up to 0.5 meter errors on one marking can be reached. In theory, to detect errors in the map, one needs a ground truth localization which is not reasonable in practice. However, by performing a first run through the mapped environment and use a Kalman smoothing scheme over the whole trajectory, estimates as close to the true states as possible can be found. A likelihood maximization based method is employed for associating the observations to the map markings making the matching process more robust. After this first run, a reliability estimate for each marking is computed and can be used to mitigate the weight of wrong mapping in the following runs.

\section{ACKNOWLEDGMENT}

This work has been carried out within SIVALab, a shared laboratory between Renault and Heudiasyc (UTC/CNRS) and in the framework of the Equipex ROBOTEX (ANR-10-EQPX44-01). It has been co-financed by the Hauts-de-France region (ERDF grant) and Renault.

\section{REFERENCES}

[1] David Betaille and Rafael Toledo-Moreo. Creating Enhanced Maps for Lane-Level Vehicle Navigation. IEEE Transactions on Intelligent Transportation Systems, 11(4):786-798, dec 2010.
[2] L. Delobel, R. Aufrã šre, C. Debain, R. Chapuis, and T. Chateau. A real-time map refinement method using a multi-sensor localization framework. IEEE Transactions on Intelligent Transportation Systems, 20(5):1644-1658, May 2019.

[3] F. Ghallabi, F. Nashashibi, G. El-Haj-Shhade, and M. Mittet. Lidarbased lane marking detection for vehicle positioning in an hd map. In 21st International Conference on Intelligent Transportation Systems (ITSC), pages 2209-2214, Nov 2018.

[4] A. Hata and D. Wolf. Road marking detection using lidar reflective intensity data and its application to vehicle localization. In 17th International IEEE Conference on Intelligent Transportation Systems (ITSC), pages 584-589, Oct 2014.

[5] Isaac Miller, Mark Campbell, and Dan Huttenlocher. Map-aided localization in sparse global positioning system environments using vision and particle filtering. Journal of Field Robotics, 28(5):619643, 2011.

[6] T. T. Nguyen, J. Spehr, M. Uhlemann, S. Zug, and R. Kruse. Learning of lane information reliability for intelligent vehicles. In IEEE International Conference on Multisensor Fusion and Integration for Intelligent Systems (MFI), pages 142-147, Sep. 2016.

[7] J. Pauls, T. Strauss, C. Hasberg, M. Lauer, and C. Stiller. Can we trust our maps? an evaluation of road changes and a dataset for map validation. In 21st International Conference on Intelligent Transportation Systems (ITSC), pages 2639-2644, Nov 2018.

[8] F. Poggenhans, N. O. Salscheider, and C. Stiller. Precise localization in high-definition road maps for urban regions. In IEEE/RSJ International Conference on Intelligent Robots and Systems (IROS), pages 21672174, Oct 2018.

[9] H. E. Rauch, C. T. Striebel, and F. Tung. Maximum likelihood estimates of linear dynamic systems. AIAA Journal, 3(8):1445-1450, August 1965.

[10] M. Schreiber, C. Knöppel, and U. Franke. Laneloc: Lane marking based localization using highly accurate maps. In IEEE Intelligent Vehicles Symposium (IV), pages 449-454, June 2013.

[11] Zui Tao, Philippe Bonnifait, Vincent Frémont, Javier Ibañez-Guzmán, and Stéphane Bonnet. Road-centred map-aided localization for driverless cars using single-frequency gnss receivers. Journal of Field Robotics, 34(5):1010-1033, 2017.

[12] R. P. D. Vivacqua, M. Bertozzi, P. Cerri, F. N. Martins, and R. F. Vassallo. Self-localization based on visual lane marking maps: An accurate low-cost approach for autonomous driving. IEEE Transactions on Intelligent Transportation Systems, 19(2):582-597, Feb 2018.

[13] L. Wei, C. Cappelle, and Y. Ruichek. Horizontal/vertical lrfs and gis maps aided vehicle localization in urban environment. In 16th International IEEE Conference on Intelligent Transportation Systems (ITSC 2013), pages 809-814, Oct 2013.

[14] A. Welte, P. Xu, and P. Bonnifait. Four-wheeled dead-reckoning model calibration using rts smoothing. In IEEE International Conference on Robotics and Automation (ICRA), volume to appear at Montreal, Canada in May 2019, 2019.

[15] Clément Zinoune, Philippe Bonnifait, and Javier Ibañez-Guzmán. A sequential test for autonomous localisation of map errors for driving assistance systems. IEEE Transactions on Intelligent Transportation Systems, pages 1377-1382, September 2012. 